\title{
ASO Author Reflections: Induced Bias Due to Crossover Within Randomized Controlled Trials in Surgical Oncology
}

\author{
George Garas, MD, $\operatorname{FRCS}^{1,2}$ \\ ${ }^{1}$ Surgical Epidemiology Unit, Department of Surgery and Cancer, Imperial College London, St. Mary's Hospital, London, \\ UK; ${ }^{2}$ Department of Surgical Research and Innovation, The Royal College of Surgeons of England, London, UK
}

\section{PAST}

Randomized controlled trials (RCTs) provide the highest level of evidence informing clinical practice; however, their implementation in surgical oncology remains challenging. ${ }^{1}$ A principal reason for this is crossover from the minimally invasive surgery (MIS) arm to the open surgery arm (open conversion) due to adverse events or difficulties experienced with the MIS-tested techniques. Crossover violates the randomization process and can lead to partial homogenization of both trial arms, with survival, the primary endpoint in surgical oncology trials, being especially affected. $^{2}$ Intention-to-treat analysis, originally developed for pharmacological trials, may not always be appropriate because, when evaluating a novel medical device or surgical technique, placebos cannot be utilized and randomization is not commonly blind. ${ }^{3}$ Crossover further adds to these drawbacks, and the implications can be profound. Clinical and cost effectiveness can be affected, which in turn may impact on decision making. The aims of this study were to identify modifiable factors associated with crossover and assess the impact of crossover on clinical endpoints, including mortality and complications.

ASO Author Reflections is a brief invited commentary on the article "Induced Bias Due to Crossover Within Randomized Controlled Trials in Surgical Oncology: A Meta-Regression Analysis of Minimally Invasive versus Open Surgery for the Treatment of Gastrointestinal Cancer" recently published in Annals of Surgical Oncology 2018;25:221-230. https://doi.org/10.1245/s10434-0176210-y.

\section{(C) The Author(s) 2018}

First Received: 9 September 2018; Published Online: 20 September 2018

G. Garas, MD, FRCS

e-mail: g.garas@imperial.ac.uk

\section{PRESENT}

The findings of this study demonstrate that crossover in surgical oncology RCTs is common, affecting one in eight patients. Moreover, its incidence was shown to reduce with increasing surgeon experience and decreasing patient comorbidity (as indicated by pretrial volume and American Society of Anesthesiologists [ASA] score, respectively). Importantly, the clinical consequences of this crossover within surgical oncology RCTs included increases in 30-day mortality, anastomotic leak rate, and complications as demonstrated by meta-regression. ${ }^{4}$ It thus becomes apparent that in the presence of crossover, an intention-totreat analysis may underestimate the underlying mortality benefit associated with MIS, i.e. the benefit that would have been observed had crossover not occurred due to partial homogenization of the study groups. Similarly, the anastomotic leak rate may be overestimated in the MIS group. Hence, in the presence of crossover, any clinical, costeffectiveness, and economic evaluation relying on traditional intention-to-treat analysis is prone to generate inaccurate results, which may impact on patient safety as well as lead to inappropriate resource allocation. ${ }^{5}$

\section{FUTURE}

Future RCTs must develop and implement strategies that include pretrial phases and surgeon credentialing by volume and/or video assessment to reduce the incidence of crossover and thus maintain randomized homogenous groups to adequately test the hypothesis. Moreover, given its independence as a predictor of crossover, as well as its potential effect on complications, it may be advisable to only include low-comorbidity patients in RCTs when initially comparing novel MIS techniques with open surgery. More complex statistical methods developed to account for the crossover effect may also need to be considered. A 
more pragmatic approach could involve reporting endpoints in three groups rather than two, i.e. the (completed) MIS, open, and converted (crossover) trial arms in addition to the traditional intention-to-treat analysis. This type of analysis will also allow the evaluation of factors associated with crossover, and thus try to predict which patients would not constitute good candidates for MIS due to their high risk of conversion and thus complications.

ACKNOWLEDGMENT Dr. George Garas, MD, FRCS, holds a Royal College of Surgeons of England Doctoral Research Fellowship (Grant Number GG 1037600/2017-2018) and is also supported by Imperial College London (Grant Number CID 337755/2015-2018) and the Alexander S. Onassis Public Benefit Foundation (Grant Number F ZM 014-1/2016-2017).

DISCLOSURE George Garas has no conflicts of any commercial interest to declare.

OPEN ACCESS This article is distributed under the terms of the Creative Commons Attribution 4.0 International License (http://crea tivecommons.org/licenses/by/4.0/), which permits unrestricted use, distribution, and reproduction in any medium, provided you give appropriate credit to the original author(s) and the source, provide a link to the Creative Commons license, and indicate if changes were made.

\section{REFERENCES}

1. Garas G, Ibrahim A, Ashrafian H, Ahmed K, Patel V, Okabayashi $\mathrm{K}$, et al. Evidence-based surgery: barriers, solutions, and the role of evidence synthesis. World J Surg. 2012;36:1723-31.

2. Rimawi M, Hilsenbeck SG. Making sense of clinical trial data: is inverse probability of censoring weighted analysis the answer to crossover bias? J Clin Oncol. 2012;30:453-8.

3. Buckley CJ, Rutherford RB, Diethrich EB, Buckley SD. Inherent problems with randomized clinical trials with observational/no treatment arms. J Vasc Surg. 2010;52:237-41.

4. Garas G, Markar SR, Malietzis G, Ashrafian H, Hanna GB, Zacharakis E, et al. Induced Bias Due to Crossover in Randomized Controlled Trials in Surgical Oncology: A Meta-regression Analysis of Minimally Invasive versus Open Surgery for the Treatment of Gastrointestinal Cancer. Ann Surg Oncol. 2018;25:221-30. https://doi.org/10.1245/s10434-017-6210-y.

5. Latimer $\mathrm{N}$. The role of treatment crossover adjustment methods in the context of economic evaluation [PhD thesis]. 2012. University of Sheffield, Sheffield, UK. 